\title{
The Mechanisms of Grain Refinement by Intensive Shearing of AZ91 Alloy Melt
}

\author{
H. Men, B. Jiang, Z Fan* \\ The EPSRC Centre for Innovative Manufacturing in Liquid Metal Engineering, BCAST (Brunel Centre \\ for Advanced Solidification Technology), Brunel University, Uxbridge, Middlesex, UB8 3PH, \\ UK
}

\begin{abstract}
It has been demonstrated recently that intensive melt shearing can be an effective approach to the grain refinement of both shape casting and continuous casting of $\mathrm{Mg}$ alloys. In the present study, the mechanisms of grain refinement by intensive melt shearing were investigated through a combination of both modeling and experimental approaches. The measurement of the cooling curves during solidification, quantification of grain size of the solidified samples and image analysis of the $\mathrm{MgO}$ particle size and size distribution in the pressurized filtration samples were performed for the AZ91 alloy with and without intensive melt shearing. The experimental results were then used as input parameters for the free growth model to investigate the mechanisms of grain refinement by intensive melt shearing. The experimental results showed that although intensive melt shearing does not change the nucleation starting temperature it increases the nucleation finishing temperature, giving rise to a reduced nucleation undercooling. The theoretical modeling using the free growth model revealed quantitatively that intensive melt shearing can effectively disperse $\mathrm{MgO}$ particles densely populated in the oxide films into more individual particles in the alloy melt, resulting in an increase of the $\mathrm{MgO}$ particle density by three orders of magnitude and the density of active nucleating $\mathrm{MgO}$ particles by a factor of 20 compared with those of the non-sheared melt. Therefore, the grain refining effect of intensive melt shearing can be confidently attributed to the significantly increased refining efficiency of the naturally occurring $\mathrm{MgO}$ particles in the alloy melt as potent nucleation sites.
\end{abstract}

Keywords: Nucleation, Oxide, Grain refinement, Magnesium alloy.

\section{Introduction}

A fine and fully equiaxed microstructure is desirable for both cast and wrought alloys since it improves the casting processes, facilitates the downstream processing and enhances the

* Corresponding author. Tel.: +44 1895 266406; fax: +44 1895269758.

E-mail address: Zhongyun.Fan@brunel.ac.uk (Z. Fan). 
mechanical properties of the as-cast components [1,2]. Grain refinement can usually be achieved through enhancing heterogeneous nucleation during solidification processing by either chemical inoculation [2,3] or physical grain refinement [4]. Chemical inoculation is achieved by addition of a specially selected chemical substance (the grain refiner) to provide exogenous crystalline particles to the alloy melt prior to solidification processing, such as $\mathrm{TiB}_{2}$ and $\mathrm{TiC}$ for $\mathrm{Al}$-alloys and $\mathrm{Zr}$ for Al-free Mg-alloys. In contrast, physical grain refinement relies on activating the endogenous crystalline particles naturally occurring in the alloy melt by a physical means, such as magnetic stirring, ultrasonic streaming and intensive melt shearing. In recent years, it has been demonstrated that intensive melt shearing provided by the MCAST process can be an effective approach for physical grain refinement [4]. The MCAST process has been developed to condition alloy melts prior to solidification processing, and solidification of the intensively sheared alloy melt results in significant grain refinement, considerable reduction of cast defects and substantial improvement of mechanical properties of the solidified materials [4-8]. For example, the grain size was reduced from $682 \mu \mathrm{m}$ without intensive melt shearing to $178 \mu \mathrm{m}$ with intensive melt shearing in the typical TP-1 test samples for the AZ91 alloy at a casting temperature of $650^{\circ} \mathrm{C}$ [4]. This process is particularly useful for grain refinement of the Albearing $\mathrm{Mg}$ alloys, for which, zirconium, as the efficient grain refiner for the Al-free $\mathrm{Mg}$ alloys, is poisoned [9] and the effective grain refiners for such alloys still remain as an active research topic [10-16]. Thus, elucidating the mechanisms of the grain refinement by intensive melt shearing is of significant importance for both scientific understanding and technological development.

For effective grain refinement, the nucleating particles need to be highly potent, i.e. with a very small nucleation barrier [2-4]. This is only possible while a lattice plane of the nucleating substrate exhibits a good atomic match with a lattice plane of the nucleated solid, and the atomic mismatch is typically less than $10 \%$ for potent particles [17]. In our previous study [4], a welldefined orientation relationship has been observed between the $\mathrm{MgO}$ and $\alpha-\mathrm{Mg}$ phases: $\left.<1 \overline{2} 10>_{\mathrm{Mg}} / /<01 \overline{1}\right\rangle_{\mathrm{MgO}}$ and $\{0002\}_{\mathrm{Mg}} / /\{111\}_{\mathrm{MgO}}$. It was found that the crystallographic misfit along the most closely packed directions $\left.\left.(<1 \overline{2} 10\rangle_{\mathrm{Mg}} / /<01 \overline{1}\right\rangle_{\mathrm{MgO}}\right)$ on the most closely packed atomic planes is only $5.46 \%$. With such potent nucleation particles, the classical spherical-cap model for heterogeneous nucleation may not be applicable [18]. The adsorption model [18, 19] and the hypernucleation theory $[20,21]$ may be more appropriate for such cases.

Quantitative prediction of nucleation events during solidification of metallic liquids is not a trivial task [22]. Maxwell and Hellawell [23] applied the classical steady state nucleation theory to calculate the number of heterogeneous nucleation events in a spatially isothermal melt as a function of the density of the nucleating particles with a single particle size. The final grain size was taken as the result of a competition between heterogeneous nucleation and growth in the melt. The nucleation rate was believed to be negligible when the temperature rises due to latent heat evolved by the growth of the new phase (recalescence) or when the nucleation sites are exhausted. Based on the Maxwell-Hellawell model, Greer et al. [24] developed the free growth model for grain initiation on potent nucleating substrates. This model suggested that new crystalline phase could start free growth, without any delay, on the potent particles at an undercooling inversely proportional to the diameter of the nucleating substrate. Unlike the Maxwell-Hellawell model, the grain initiation in the free growth model is neither time-dependent nor stochastic, and a distribution of particle sizes was applied rather than a single size. The free 
growth model revealed that the largest particles in the melt start to grow first as soon as the required undercooling is reached, followed by the progressively smaller ones as the undercooling is increased. The grain sizes are limited by the recalescence, after which no further grain initiation occurs. With size distribution of the particles as input parameters, the free growth model has been successfully applied in the cases of aluminum alloys inoculated by the Al-Ti-B and Al-Ti-C based grain refiners [24, 25] and magnesium alloy by the SiC refiner [26].

The objective of the present study is to elucidate the mechanisms of grain refinement by intensive melt shearing through the combination of theoretical modeling based on the free growth model and the experimental investigation. We apply the free growth model to quantitatively study the initial stage of solidification in the sheared and non-sheared AZ91 alloy melt. The modeling results, combined with the experimental observations, are used to understand the roles of the $\mathrm{MgO}$ particles in grain initiation during solidification with and without prior intensive melt shearing.

\section{Experimental and modeling procedures}

\subsection{Cooling curves during solidification and the resultant microstructures}

Fig.1 shows a schematic illustration of the experimental setup for measuring the cooling curves during solidification of the AZ91 alloy melt. The inner steel crucible, covered with a $13 \mathrm{~mm}$ thick layer of thermal insulation, was placed in the centre of an outer crucible to ensure a consistent cooling condition by minimizing the influence of the environment. The AZ91 alloy ingot $(\mathrm{Mg}-$ 8.6Al-0.67Zn-0.22Mn, all in wt.\%) was melted at $680^{\circ} \mathrm{C}$ under a protective gas mixture $\left(\mathrm{N}_{2}\right.$ containing 0.4 vol.\% $\mathrm{SF}_{6}$ ). The alloy melt was then transferred to the MCAST unit and subjected to intensive shearing at $800 \mathrm{rpm}$ for $45 \mathrm{~s}$ at $650^{\circ} \mathrm{C}$. The alloy melt with or without melt shearing was poured into the inner crucible pre-heated to $650^{\circ} \mathrm{C}$, and was immediately covered by a thin layer of salt flux and a steel lid to protect the melt from oxidation. A $\Phi 0.5 \mathrm{~mm} \mathrm{~K}$-type thermocouple supported by a $\Phi 3 \mathrm{~mm}$ steel tube fixed on the lid was located in the center of the inner crucible to measure the melt temperature. The melt temperature was recorded through a data logger with a frequency of $20 \mathrm{HZ}$.

Samples for microstructural analysis were taken from the central position (the position of the thermocouple tip) of the solidified ingot. The microstructures were quantified to obtain the grain sizes of both sheared and non-sheared samples. Metallographic sections for optical microscopy (OM) were prepared using standard metallographic procedures, followed by color etching [27].

The grain size is the value of mean linear intercept length, $\bar{l}$. The number of grains per unit volume, $N_{\mathrm{v}}$, is obtained using the following equation [24]:

$$
N_{\mathrm{v}}=\frac{0.5}{\bar{l}^{3}}
$$

where $N_{\mathrm{v}}$ is taken as the lower bound of the number density of the active nucleating particles in the melt, and is used as the number density of active nucleating particles, $N_{\mathrm{e}}$.

\subsection{Measuring the MgO particles size distribution}


The size distribution of $\mathrm{MgO}$ particles in the sheared melt was obtained by analyzing the pressurized filtration samples with intensive melt shearing. The oxide and intermetallic inclusions in the sheared AZ91 melt were collected by the pressurized filtration technique, as described in detail elsewhere [4]. The solidified material adjacent to the filter with a high concentration of inclusion particles was sectioned and prepared for detailed metallographic examination. Metallographic sections for scanning electron microscopy (SEM) were prepared using standard metallographic procedure. The SEM examination was carried out using a Zeiss Supera 35 FEG microscope, equipped with an energy dispersive spectroscopy (EDS) facility, operated at an accelerating voltage of $5-15 \mathrm{kV}$. Image analysis was used to obtain the size distribution of $\mathrm{MgO}$ particles from the SEM micrographs.

\subsection{Modeling method}

The particle size distribution is a crucial factor in nucleation analysis. Turnbull [28] derived the size distribution of nucleating patches by fitting the data on solidified fraction of the undercooled liquid mercury droplets. Greer et al. [24] reversed this procedure by using the size distribution of nucleating particles $\left(\mathrm{TiB}_{2}\right)$ determined experimentally from the grain refiner alloy $(\mathrm{Al}-5 \mathrm{Ti}-1 \mathrm{~B}$, all in wt.\%) to predict quantitatively the grain size of the inoculated aluminum alloys. The population distribution of the $\mathrm{TiB}_{2}$ particles was found to follow a log-normal function [25]:

$$
N(\mathrm{~d})=\frac{N_{0}}{\sigma d \sqrt{2 \pi}} \exp -\left(\frac{\left.\mathbf{h}(d)-\ln \left(d_{0}\right)^{2}\right]}{2 \sigma^{2}}\right)
$$

where $d_{0}$ is the geometric mean diameter, $\sigma$ the geometric standard deviation, $N(d)$ the number of particles of diameter between $d$ and $d+\Delta d$ and $N_{0}$ the total number of particles. The required undercooling for free growth on a given particle, $\Delta T_{\mathrm{fg}}$, is simply related to its diameter, $d$, by [24]

$$
\Delta T_{\mathrm{fg}}=\frac{4 \gamma}{\Delta S_{\mathrm{v}} d}
$$

where $\gamma$ is the solid-liquid interfacial energy and $\Delta S_{\mathrm{v}}$ is the entropy of fusion per unit volume.

However, the approach adopted by Greer et al. to obtain particle size distribution cannot be applied in the case of physical grain refinement. Here we propose a different approach to obtain the size distribution of $\mathrm{MgO}$ particles in the intensively sheared alloy melt. For convenience, the grain initiation will be referred to as nucleation from now on. Firstly, the maximum undercoolings of the sheared and non-sheared AZ91 alloy melt were obtained from the measured cooling curves. In the present study, the temperature at which the cooling curve starts to deviate from the linearity is defined as the nucleation starting temperature, $T_{\mathrm{s}}$, and the minimum temperature before recalescence as the nucleation finishing temperature, $T_{\mathrm{f}}$. The temperature interval between the alloy liquidus $\left(T_{1}\right)$ and $T_{\mathrm{f}}$ is taken as the maximum undercooling for nucleation, $\Delta T_{\max }$, i.e. the undercooling required for the smallest particles which have participated in the nucleation process. In addition, the diameter of the smallest active particles is defined as the critical diameter, $d_{\mathrm{c}}$. According to equation (3), $d_{\mathrm{c}}$ can be determined from $\Delta T_{\max }$ of the melt. Secondly, the number density of the active nucleating particles was estimated from the grain size of the corresponding solidified samples according to equation (1). Thirdly, the size distribution $\left(\sigma\right.$ and $\left.d_{0}\right)$ of $\mathrm{MgO}$ particles in the sheared melt was obtained from the image 
analysis of the $\mathrm{MgO}$ particles in the pressurized filtration samples. The integration of $N(\mathrm{~d})$ from $d_{\mathrm{c}}$ to the maximum particle size, $d_{\max }$, provides the number density of active nucleating particles, $N_{\mathrm{e}}$. The minimum particle size used in this modeling is denoted as $d_{\min }$. Therefore, the total number of $\mathrm{MgO}$ particles, $N_{0}$, and then the population density, $N(\mathrm{~d})$ (from $d_{\min }$ to $d_{\max }$ ), can be obtained through applying equation (2) with $N_{\mathrm{e}}, d_{\mathrm{c}}, \sigma$ and $d_{0}$ as input parameters.

After the particle size distribution is obtained, we followed closely the treatment used for grain initiation by Greer et al. [24, 25]. However, a dendritic growth model instead of a spherical growth model was applied in the present modeling. Dendrites can grow with a tip undercooling $\Delta T$, which depends on growth velocity $V$, temperature gradient $G$ and alloy composition $C_{0}$ [29]. Empirically, it was found [30, 31] that

$$
\Delta T=\frac{G D}{V}+\left(\frac{C_{0} V}{A}\right)^{1 / 2}
$$

with

$$
A=\frac{D}{8 m(1-k) \Gamma}
$$

where $D$ is the self-diffusion coefficient in liquid, $m$ the liquidus slope, $k$ the distribution coefficient and $\Gamma$ the Gibbs-Thomson parameter,

$$
\Gamma=\frac{\gamma}{\Delta S_{\mathrm{v}}} .
$$

Under the assumption of an isothermal melt, the growth velocity of the dendrite tip can be given as

$$
V=\frac{A \Delta T^{2}}{C_{0}} .
$$

The numerical calculation was implemented in Visual Basic 6.0, and the undercooling increment between steps was typically $0.01 \mathrm{~K}$. All the relevant parameters and their typical values used in the modeling are listed in Table 1.

\section{Results}

\subsection{Measured cooling curve and grain size}

Fig. 2 shows the experimentally determined cooling curves for the AZ91 alloy melt with and without intensive melt shearing. Near the liquidus $\left(T_{1}\right)$, the typical cooling rate is about $0.22^{\circ} \mathrm{C} / \mathrm{s}$ in the sheared melt, and about $0.2^{\circ} \mathrm{C} / \mathrm{s}$ in the non-sheared melt. Both of the cooling curves showed pronounced recalescence, agreeing with the basic feature of the free growth model [24]. Because it is not possible to obtain an accurate value of $T_{1}$ from the measured cooling curve, the experimental $\Delta T_{\max }$ is approximately determined relative to the thermal plateau temperature after 
recalescence, $T_{\mathrm{r}}$. Usually the value of $T_{1}-T_{\mathrm{r}}$ is small, corresponding to the required undercooling for crystal growth during solidification. Therefore, the value of $T_{\mathrm{r}}-T_{\mathrm{f}}$ is used as a good approximation of $\Delta T_{\max }$, which is marked in Fig. 2 and 3. The measured $\Delta T_{\max }$ is $0.62^{\circ} \mathrm{C}$ with intensive melt shearing and $0.98^{\circ} \mathrm{C}$ without intensive melt shearing. From equation (3), $d_{\mathrm{c}}$ was then determined to be $0.95 \mu \mathrm{m}$ with intensive melt shearing and $0.60 \mu \mathrm{m}$ without intensive melt shearing.

Fig. 3 shows a schematic illustration of a typical cooling curve, the first derivative $(\partial T / \partial t)$ and the second derivative $\left(\partial^{2} T / \partial t^{2}\right)$ of the cooling curve following the treatment used in Ref $[33,34]$. It is almost impossible to determine $T_{\mathrm{s}}$ from the original cooling curve, but $T_{\mathrm{s}}, T_{\mathrm{f}}$ and $T_{\mathrm{r}}$ can be easily identified in the first and second derivative of the cooling curves. The average values and the corresponding standard deviations of the measured $T_{\mathrm{s}}, T_{\mathrm{f}}$ and $T_{\mathrm{r}}$ from nine original cooling curves (five for non-sheared melt and four for sheared melt) are presented in Table 2. Firstly, the values for $T_{\mathrm{s}}$ are almost identical for the non-sheared and sheared melts, indicating that the size of the largest particle that triggers nucleation is the same for both the sheared and non-sheared melts; secondly, the value of $T_{\mathrm{f}}$ in the sheared melt is $0.4^{\circ} \mathrm{C}$ higher than that in the non-sheared melt, suggesting that intensive melt shearing decreases the maximum nucleation undercooling $\left(\Delta T_{\max }\right)$ and gives a larger critical size for nucleation $\left(d_{\mathrm{c}}\right)$; thirdly, $T_{\mathrm{r}}$ is not changed by intensive melt shearing, implying that intensive melt shearing does not change the growth temperature. This confirms for the first time that intensive melt shearing only disperses particle agglomerates into individual particles without breaking up the larger particles into smaller ones. This means that intensive melt shearing only promotes a uniform spatial distribution of the $\mathrm{MgO}$ particles in the entire volume of the alloy melt without changing the particle size and size distribution.

The average grain size of the solidified samples was determined to be $2005 \mu \mathrm{m}$ for the nonsheared samples and $1210 \mu \mathrm{m}$ for the sheared samples. According to equation (1), the number density of active nucleating particles $\left(N_{\mathrm{e}}\right)$ is determined to be $0.35 \mathrm{~mm}^{-3}$ and $0.06 \mathrm{~mm}^{-3}$ for the cases with and without melt shearing, respectively. Therefore, it can be concluded that intensive melt shearing increases the number density of the active nucleating particles.

\subsection{MgO particles size distribution in the sheared alloy melt}

Fig. 4a shows a SEM micrograph of the $\mathrm{MgO}$ oxides collected at $650^{\circ} \mathrm{C}$ by the pressurized filtration of the non-sheared AZ91 alloy melt. It indicates that the oxide films consist of fine $\mathrm{MgO}$ particles densely populated in a liquid matrix. Fig. 4(b) shows the MgO particles in the samples filtered from the sheared alloy melt. It can be seen that the oxide films have been significantly disintegrated into more individual particles. This suggests that the fine $\mathrm{MgO}$ particles in the oxide films are uniformly dispersed into the alloy melt by intensive shearing, so that each particle could serve as a potential nucleating site during solidification.

Fig. 5 shows the measured size distribution of $\mathrm{MgO}$ particles in the filtered sample from the sheared alloy melt. The total number of particles used for obtaining the distribution was in the order of 2000. The size distribution can be well fitted by equation (2), and the curve in the figure is indicative of the fitted $\log$-normal function with $d_{0}=0.07 \mu \mathrm{m}$ and $\sigma=0.45$.

\subsection{Population distribution of $\mathrm{MgO}$ particles}


The total number of $\mathrm{MgO}$ particles $\left(N_{0}\right)$ can be calculated from equation (2) with $\sigma, d_{0}, N_{\mathrm{e}}$ and $d_{\mathrm{c}}$ as input parameters. $N_{0}$ was determined to be $3.2 \times 10^{8} \mathrm{~mm}^{-3}$ and $2.1 \times 10^{5} \mathrm{~mm}^{-3}$ for the sheared non-sheared alloy melt, respectively. It is interesting to note that the value of $N_{0}$ for the nonsheared melt is very close to the total number $\left(5 \times 10^{4} \mathrm{~mm}^{-3}\right)$ of $\mathrm{TiB}_{2}$ particles with the addition of $1 \mathrm{ppt}$ Al-5Ti-1B grain refiner [24]. This indicates that as far as heterogeneous nucleation is concerned, there are sufficient numbers of naturally occurring $\mathrm{MgO}$ particles in the alloy melt prepared under the standard melting conditions. The purpose of physical grain refining is to increase the efficiency of such endogenous particles for enhanced heterogeneous nucleation.

The $\mathrm{MgO}$ population density ( $N(\mathrm{~d})$ ) can then be obtained from equation (2) using $d_{\min }, d_{\max }, \sigma, d_{0}$ and $N_{0}$ as input parameters. Fig. 6a shows the calculated $N(\mathrm{~d})$ for the $\mathrm{MgO}$ particles as a function of $d$ in the sheared melt using $\sigma=0.45, d_{0}=0.07 \mu \mathrm{m}, d_{\min }=0.01 \mu \mathrm{m}$ and $d_{\max }=6.0 \mu \mathrm{m}$. Repeated calculations using different sets of $d_{\min }$ and $d_{\max }$ have shown that the range of particle sizes used for the calculations has little effect on the resultant population density. Since intensive melt shearing only disperse $\mathrm{MgO}$ particles agglomerates into individual particles without changing the actual size of individual particles as discussed in Section 3.1, the size distribution ( $\sigma$ and $d_{0}$ ) of $\mathrm{MgO}$ particles in the non-sheared melt is assumed to be the same as that in the sheared melt. Fig. $6 \mathrm{~b}$ shows the calculated $N(\mathrm{~d})$ as a function of $d$ for the non-sheared melt. Comparing Fig. 6a with Fig. 6b, it can be concluded that the density of the potential nucleating sites in the sheared melt is about 3 orders of magnitude higher than that in the non-sheared melt, showing clearly the power of intensive melt shearing.

\subsection{Validation of the free growth model}

Once all the necessary parameters are in place, it is possible to apply the free growth model to predict the solidification process and the resultant microstructures under different solidification conditions, and then to compare the model predictions with the experimental results as a direct validation of the free growth model. Fig. 7 shows the simulated cooling curves with and without intensive melt shearing. The cooling rate used in the simulation is $0.22^{\circ} \mathrm{C} / \mathrm{s}$ with intensive melt shearing, and $0.2^{\circ} \mathrm{C} / \mathrm{s}$ without intensive melt shearing, which are the same as the experimental conditions. The calculated $\Delta T_{\max }$ is $0.71^{\circ} \mathrm{C}$ with intensive melt shearing, and $0.99^{\circ} \mathrm{C}$ without intensive melt shearing. The grain size predicted by the model is $0.671 \mathrm{~mm}$ with intensive melt shearing and $1.931 \mathrm{~mm}$ without intensive melt shearing. The theoretical predictions (Fig. 7) are in good agreement with the experimental results (Fig. 2) in both sheared and non-sheared cases.

Fig. 8 shows the theoretically predicted nucleation finishing temperatures $\left(T_{\mathrm{f}}\right)$ and the solid fraction at $T_{\mathrm{f}}$ as a function of cooling rate. Fig. 8a reveals that $T_{\mathrm{f}}$ decreases with the increase of cooling rate although the predicted $T_{\mathrm{f}}$ for the sheared melt is always higher than that for the nonsheared melt at the same cooling rate, which is consistent with the experimental observations (Fig. 2). Fig. $8 \mathrm{~b}$ shows that the solid fraction at $T_{\mathrm{f}}$ increases with the increase of cooling rate, and that at a cooling rate of $0.2^{\circ} \mathrm{C} / \mathrm{s}$, the predicted solid fraction at $T_{\mathrm{f}}$ for the non-sheared melt is $0.024 \%$, which is greater than $0.015 \%$ predicted for the sheared melt. It is interesting to note that the predicted solid fraction at $T_{\mathrm{f}}$ has the same order of magnitude as that obtained by Greer et al in the inoculated $\mathrm{Al}$ melt (about 0.02\%) [25]. At $T_{\mathrm{f}}$, the latent heat released from the growing solid phase is balanced by the heat extraction from the solidification environment, and further growth of the solid phase will cause recalescence. Therefore, increasing the cooling rate reduces the nucleation finishing temperature $T_{\mathrm{f}}$, which will, in turn, increase the solid fraction at $T_{\mathrm{f}}$. 
The good agreement between the theoretical predictions and the experimental observations suggests that the free growth model can be applied reliably in both sheared and non-sheared cases to investigate the grain refining mechanisms of AZ91 alloy with intensive melt shearing.

\subsection{Mechanisms of grain refinement by intensive melt shearing}

Fig. 9a shows the theoretically predicted number density of active nucleating particles as a function of cooling rate. Although the number of active particles increases with the increase of cooling rate in both the sheared and non-sheared cases, the increase in the sheared melt is much more pronounced than that in the non-sheared melt. For instance, at a cooling rate of $0.2^{\circ} \mathrm{C} / \mathrm{s}$, the number density of active particles is $1.5 \mathrm{~mm}^{-3}$ and $0.07 \mathrm{~mm}^{-3}$ for the cases with and without intensive melt shearing, respectively. This means that the number density of active particles is increased by a factor of 20 by the intensive melt shearing. Table 3 summarizes the physical parameters extracted by the theoretical analysis.

The dramatic increase in the number density of active nucleating particles can be attributed to the dispersive function of intensive melt shearing. In the non-sheared melt, the nucleation of the $\alpha$ $\mathrm{Mg}$ phase first occurs on the largest $\mathrm{MgO}$ particle available in the oxide film, and the smaller particles in the same oxide film will be made redundant due to the local temperature rise caused by the release of latent heat, resulting in the complete engulfment of the rest of the particles in the growing solid phase. The number of active particles in the non-sheared melt is, therefore, determined by the number of the oxide films, which can only act as single entities for heterogeneous nucleation. This argument is supported by the fact that the resultant average grain size $(2 \mathrm{~mm})$ is much larger than the size of the largest oxide film (about a few hundred microns) [4]. With intensive melt shearing, the $\mathrm{MgO}$ particles in the oxide films were effectively dispersed into a greater number of individual particles, resulting in an increase of three orders of magnitude in the total number of $\mathrm{MgO}$ particles in the sheared melt as presented previously. According to equation (2), the number of active particles in the sheared melt would be expected to be in the same order of magnitude at the same $\Delta T_{\max }$. However, $T_{\mathrm{f}}$ in the sheared melt is higher than that in the non-sheared melt at the same cooling rate (Fig. 8a), and hence $\Delta T_{\max }$ of the sheared melt is smaller than that of the non-sheared melt. As a consequence, the effective increase of active particles in the sheared melt is only by a factor of 20, compared to that in the non-sheared melt. This substantial increase in the number of active nucleating particles by intensive melt shearing will naturally lead to a significant reduction in grain size, as shown in Fig. 9b. At a cooling rate of $0.2^{\circ} \mathrm{C} / \mathrm{s}$, the predicted grain size is reduced from $1.931 \mathrm{~mm}$ for the non-sheared melt to $0.691 \mathrm{~mm}$ for the sheared melt. Furthermore, Fig. 9b shows that the grain refining effect by intensive melt shearing is more pronounced at low cooling rates than that at high cooling rates.

\section{Discussion}

Due to the assumption of an isothermal melt, the free growth model is usually applicable for a small volume of melt solidifying under a low cooling rate, such as in the case of the standard TP1 test with a cooling rate of about $3.5 \mathrm{~K} / \mathrm{s}$ and a melt volume of about $100 \mathrm{~cm}^{3}$ [35]. In the present experiments, the maximum volume of the melt is $157 \mathrm{~cm}^{3}$, and the measured cooling rate is around $0.22^{\circ} \mathrm{C} / \mathrm{s}$ at temperatures close to the alloy liquidus. Such solidification conditions can be regarded, to a reasonably good approximation, as being isothermal. This justifies the application 
of the free growth model for the simulation of the solidification process under the current experimental conditions.

The free growth model requires that the particles are potent for heterogeneous nucleation. Generally, the $\Delta T_{\max }$ is small for potent nucleating particles, and increases with the reduction of the average size of the active nucleating particles according to equation (3). Equation (3) holds true at temperatures near $T_{1}$, and may become less valid with increasing $\Delta T[36,37]$. Thus, the relatively small particles used in this modeling may lead to some degree of uncertainty in the simulation results. In addition, the cooling rate, as an input parameter, has a significant affect on the simulation results, as shown in Fig. 8a and Fig. 9b. The cooling rates used in the modeling according to the measured cooling curves may also lead to a minor discrepancy between the experimental and the simulation results. All these factors contribute to the discrepancy between the simulation results and the experimental observations. However, the theoretically predicted cooling curve and grain size of the solidified samples exhibit good agreement with the experimental results for both the non-sheared and sheared melts, supporting the validity of using the current modeling on the free growth model. Therefore, the free growth model can be applied with great confidence to quantitatively study the solidification process in the intensively sheared melt under quasi-isothermal conditions.

Grain refinement by intensive melt shearing derives primarily from the effective dispersion of the $\mathrm{MgO}$ particles in the naturally occurring oxide films into fine dispersion of individual particles in the melt. Since the MgO particles are loosely packed (although densely populated) inside the oxide films, dispersion of such oxide particles can be achieved relatively easily if the shear rate and the shearing time are beyond a critical level [4]. Once all the MgO particles in the oxide films have been completely dispersed into individual particles, further increase of shearing will not achieve further grain refinement. In addition, the solubility of oxygen in the $\mathrm{Mg}$-alloy melt is very low, suggesting that neither could the largest particles grow nor could the smallest particles dissolve in the melt during intensive melt shearing. Therefore, both the size and size distribution of the $\mathrm{MgO}$ particles will remain unchanged during intensive melt shearing.

Intensive melt shearing led to an increase in the total number of $\mathrm{MgO}$ particles in the sheared melt by three orders of magnitude compared with that in the non-sheared melt. As a consequence, the density of active $\mathrm{MgO}$ particles in the sheared melt is increased by a factor of 20 under the same solidification conditions. Thus, the grain size with melt shearing becomes considerably finer than that without melt shearing. The efficiency of the nucleating particles can be defined as the ratio of the number of active nucleating particles to the total number of potential nucleating particles. As mentioned previously, the total number of $\mathrm{MgO}$ particles does not change during intensive melt shearing while the number of active particles increases dramatically. Consequently, the efficiency of the $\mathrm{MgO}$ particles in the sheared melt is much higher than that in the non-sheared melt. The significant improvement in the efficiency of $\mathrm{MgO}$ particles leads to the effective grain refining in the sheared melt.

\section{Summary}

In this work we have investigated the grain refining mechanisms by intensive melt shearing prior to solidification processing through coupling of the experimental and modeling approaches. The 
experimental approach was used to obtain cooling curves during solidification under quasiisothermal conditions, the $\mathrm{MgO}$ particle size and size distribution in the alloy melt and the resultant grain size in the solidified samples. Experimental results were then used as input parameters for the free growth model to quantitatively analyze the nucleation behavior of AZ91 alloy melt with and without melt shearing.

The experimental results showed that although intensive melt shearing does not change the nucleation starting temperature it does increase the nucleation finishing temperature and consequently decreases the maximum nucleation undercooling. The experimental results also revealed that the total number of $\mathrm{MgO}$ particles in the AZ91 alloy melt is $2.1 \times 10^{5} \mathrm{~mm}^{-3}$, which is comparable with that of $\mathrm{TiB}_{2}$ particles with the addition of $1 \mathrm{ppt}$ Al-5Ti-1B grain refiner $\left(5 \times 10^{4} \mathrm{~mm}^{-3}\right)$, confirming that there are sufficient endogenous particles in the non-sheared alloy melt as potential heterogeneous nucleation sites. Intensive melt shearing can increase the $\mathrm{MgO}$ number density to $3.2 \times 10^{8} \mathrm{~mm}^{-3}$, which is about three orders of magnitude higher than that in the non-sheared melt. It was found that the density of the active $\mathrm{MgO}$ particles in the sheared melt is about 20 times higher than that in the non-sheared melt, giving rise to a significant grain refinement.

The free growth model has been successfully applied to quantitatively study the nucleation process in both the sheared and non-sheared AZ91 alloy melt, with the size distribution of $\mathrm{MgO}$ particles as an input parameter. The calculated cooling curves and grain sizes are in good agreement with the experimental results. The mechanisms of grain refinement in the intensively sheared AZ91 alloy melt were investigated according to the modeling results and the experimental observations. It has been shown quantitatively that the $\mathrm{MgO}$ particles in the oxide films were effectively dispersed into more individual particles by intensive melt shearing. This will significantly increase the number density of the active nucleant particles in the sheared melt, and consequently significant grain refinement.

\section{Acknowledgements}

The authors wish to thank Dr Y. Zuo and Mr P. Enright for their help with the cooling curve experiments. Financial support under grant EP/H026177/1 from the EPSRC is gratefully acknowledged.

\section{References:}

1. Granger DA. In: Welch BJ, editor. Light Metals 1998. Warrendale (PA): TMS; 1998. p. 94152.

2. McCartney DG. Int Mater Rev 1989;34:247.

3. StJohn DH, Qian M, Easton MA, Cao P, Hildebrand Z. Metall Mater Trans A 2005;36:1669.

4. Fan Z, Wang Y, Xia M, Arumuganathar S. Acta Mater 2009;57:4891.

5. Fan Z, Liu G, Wang Y. J Mater Sci 2006;41:3631.

6. Wang Y, Liu G, Fan Z. Acta Mater 2006;54:689.

7. Hitchcock M, Wang Y, Fan Z. Acta Mater 2007;55:1598. 
8. Bayandorian I, Bian Z, Xia M, Zhang H, Scamans, Fan Z. In: Nyberg NA, Agnew SR, Neelameggham NR, Pekguleryuz MO, editors. Magnesium technology 2009. San Francisco: TMS; 2009. p. 363-68.

9. Emley EF. In: Principles of magnesium technology. Oxford: Pergamon Press; 1966. P. 12656.

10. Cao P, Qian M, StJohn DH. Scripta Mater 2005;53:841.

11. Farbenindustrie IG, British Patent GB359425, 1931.

12. Byun JY, Kwon SI, Ha HP, Yoon JK. In: Kainer KU, editor. Magnesium alloys and their applications. Weinheim: Wiley-VCH; 2003. P. 713-8.

13. Kim YM, Yim CD, You BS. Scripta Mater 2007;57:691.

14. Cao P, Qian M, StJohn DH. Scripta Mater 2004;51:125.

15. Nelson CE. Trans AFS 1948;56:1.

16. Tiner N. AIME Tech Pub 1945;12:1.

17. Zhang MX, Kelly PM, Qian M, Taylor JA. Acta Mater 2005;53:3261.

18. Kim WT, Cantor B. Acta Metall Mater 1994;42:3115.

19. O’Reilly KAQ, Cantor B. Acta Metal 1995;43:405.

20. Jones GP, Pearson J. Metall Trans B 1976;7:223.

21. Jones GP. In: Beech J, Jones H, editors. Solidification processing 1987. London: The Institute of Metals; 1988. p.496-9.

22. Donnelly SE, Birtcher RC, Allen CW, Morrison I, Furuya K, Song MH, et al. Science 2002;296:507.

23. Maxwell I, Hellawell A. Acta Metall 1975;23:229.

24. Greer AL, Bunn AM, Tronche A, Evans PV, Bristow DJ. Acta Mater 2000;48:2823.

25. Quested TE, Greer AL. Acta Mater 2004;52:3859.

26. Günther R, Hartig Ch, Bormann R. Acta Mater 2006;54:5591.

27. Maltais A, Dubé D, Fiset M, Laroche G, Turgeon S. Mater. Characterization 2004;52:103.

28. Turnbull D. Acta Metall 1953;1:8.

29. Hunt JD. Mater Sci Engi 1984;65:75.

30. Burden MH, Hunt JD. J Cryst Growth 1974;22:99.

31. Tassa M, Hunt JD. J Cryst Growth 1976;34:38.

32. Avedesian M, Baker H. In: 1999 Magnesium and magnesium alloys. Materials Park, $\mathrm{OH}$ : ASM; 1999. p. 231-33.

33. Nafisi S, Ghomashchi R. Mater Sci Eng A 2007;452-453:445.

34. Backerud L, Chai G, Tamminen J. In: Solidification characteristics of aluminum alloys, Foundry alloys. Skanaluminum: AFS; 1990, p. 3-7.

35. Quested TE, Greer AL. Acta Mater 2005;53:4643.

36. Christian JW. In: The theory of transformations in the metals and alloys, $2^{\text {nd }}$ ed. Oxford: Pergamon press; 1975. p. 422-79.

37. Reed-Hill RE, Abbaschian R. In: Physical metallurgy principles, $3^{\text {rd }}$ ed. Boston: PWS-KENT; 1992. p. 479-514. 
Tables:

Table 1 Parameters used in the calculations.

\begin{tabular}{lcccc}
\hline Quantity & Symbol & Units & Value & Ref. \\
\hline Gibbs-Thomson parameter & $\Gamma$ & $\mathrm{Km}$ & $1.48 \times 10^{-7}$ & {$[32]$} \\
Enthalpy of fusion per unit volume & $\Delta H_{\mathrm{v}}$ & $\mathrm{J} / \mathrm{m}^{3}$ & $6.75 \times 10^{8}$ & {$[32]$} \\
Heat capacity of melt per unit volume & $C_{\mathrm{v}}$ & $\mathrm{J} / \mathrm{Km}^{3}$ & $2.59 \times 10^{6}$ & {$[32]$} \\
Diffusivity in melt (Al in $\mathrm{Mg})$ & $D$ & $\mathrm{~m}^{2} / \mathrm{s}$ & $2.7 \times 10^{-9}$ & {$[32]$} \\
Liquidus slope & $m$ & $\mathrm{~K} / \mathrm{wt} \%$ & -6.87 & {$[3]$} \\
Equilibrium partition coefficient & $k$ & & 0.37 & {$[3]$} \\
\hline
\end{tabular}

Table 2 Measured $T_{\mathrm{s}}, T_{\mathrm{f}}, T_{\mathrm{r}}$ and $\Delta T_{\max }$ from the cooling curves of the melts and grain sizes from the solidified samples.

\begin{tabular}{lccccc}
\hline Samples & $T_{\mathrm{s}}\left({ }^{\circ} \mathrm{C}\right)$ & $T_{\mathrm{f}}\left({ }^{\circ} \mathrm{C}\right)$ & $T_{\mathrm{r}}\left({ }^{\circ} \mathrm{C}\right)$ & $\Delta T_{\max }\left({ }^{\circ} \mathrm{C}\right)$ & Grain sizes $(\mu \mathrm{m})$ \\
\hline $\begin{array}{l}\text { Without } \\
\text { shearing }\end{array}$ & $597.97 \pm 0.07$ & $597.05 \pm 0.12$ & $598.15 \pm 0.12$ & $0.98 \pm 0.14$ & 2005 \\
With shearing & $597.91 \pm 0.12$ & $597.47 \pm 0.10$ & $598.15 \pm 0.12$ & $0.62 \pm 0.07$ & 1210 \\
\hline
\end{tabular}

Table 3 Physical parameters extracted by theoretical analysis at a cooling rate of $0.2^{\circ} \mathrm{C} / \mathrm{s}$

\begin{tabular}{lcccccc}
\hline Samples & $d_{\mathrm{c}}(\mu \mathrm{m})$ & $d_{0}(\mu \mathrm{m})$ & $\sigma$ & $N_{0}\left(\mathrm{~mm}^{-3}\right)$ & $N_{\mathrm{e}}\left(\mathrm{mm}^{-3}\right)$ & Solid fraction at $T_{\mathrm{f}}(\%)$ \\
\hline $\begin{array}{l}\text { Without } \\
\text { shearing }\end{array}$ & 0.60 & 0.07 & 0.45 & $2.1 \times 10^{5}$ & 0.07 & 0.024 \\
$\begin{array}{l}\text { With shearing } \\
\text { W }\end{array}$ & 0.95 & 0.07 & 0.45 & $3.2 \times 10^{8}$ & 1.5 & 0.015 \\
\hline
\end{tabular}




\section{Figure captions:}

Fig. 1 Schematic illustration of the experimental setup for measuring the cooling curves of the AZ91 alloy melt with and without prior intensive melt shearing. The inner steel crucible, covered by a $13 \mathrm{~mm}$ thick thermal insulation, was placed in the center of a larger outer crucible to ensure a consistent cooling condition. The alloy melt with or without prior intensive melt shearing was poured into the inner crucible, and covered immediately by a thin layer of salt flux and a steel lid to prevent oxidation. A K-type thermocouple with a diameter of $0.5 \mathrm{~mm}$ supported by a steel tube was located in the center of the inner crucible to measure the melt temperature, and temperature data was recorded at a frequency of $20 \mathrm{HZ}$.

Fig. 2 Measured cooling curves for the AZ91 alloy melt with and without prior melt shearing. Both the cooling curves exhibit pronounced recalescence. The maximum undercooling $\Delta T_{\max }$ is estimated on the cooling curve relative to the thermal plateau after recalescence. $\Delta T_{\max }$ is $0.62^{\circ} \mathrm{C}$ for the sheared melt and $0.98^{\circ} \mathrm{C}$ for the non-sheared melt. The measured cooling rate near $T_{1}$ is $0.22^{\circ} \mathrm{C} / \mathrm{s}$ for the sheared melt and $0.2^{\circ} \mathrm{C} / \mathrm{s}$ for the non-sheared melt.

Fig. 3 Schematic illustrations of (a) the cooling curve, (b) the first derivative $(\partial T / \partial t)$ and (c) the second derivative $\left(\partial^{2} T / \partial t^{2}\right)$ of the original cooling curve. $T_{\mathrm{f}}$ and $T_{\mathrm{r}}$ are determined from the points at $\partial T / \partial t=0$ in (b) and $T_{\mathrm{s}}$ is determined from the point at $\partial^{2} T / \partial t^{2}=0$ in (c).

Fig. 4 SEM micrographs showing the morphology of the $\mathrm{MgO}$ particles collected at $650^{\circ} \mathrm{C}$ by the pressurized filtration of (a) the non-sheared and (b) the sheared AZ91 alloy melt. The micrographs indicate that the oxide films in the non-sheared sample consist of fine $\mathrm{MgO}$ particles densely populated in the liquid Mg matrix. Such oxide films have been significantly dispersed by intensive melt shearing into more individual particles. The alloy melt was sheared at $650^{\circ} \mathrm{C}$ and $800 \mathrm{rpm}$ for $45 \mathrm{~s}$ before filtration.

Fig. 5 The measured size distribution of the MgO particles in the sheared AZ91 alloy melt collected by the pressurized filtration. Image analysis of SEM micrographs was used to obtain the size distribution, and the total number of particles used for this analysis was in the order of 2000. The size distribution of $\mathrm{MgO}$ particles can be well fitted by a log-normal function, as indicated by the solid curve. The log-normal fit gives $d_{0}=0.07 \mu \mathrm{m}$ and $\sigma=0.45$.

Fig. 6 The calculated population densities of the potential nucleating particles as a function of the particle diameter $d$ in (a) the sheared and (b) non-sheared melt. Log-normal distributions of particles with $\sigma=0.45$ and $d_{0}=0.07 \mu \mathrm{m}$ are assumed in both the cases. It can be seen that the density of $\mathrm{MgO}$ particles in the sheared melt is three orders of magnitude higher than that in the non-sheared melt.

Fig. 7 The theoretically predicted cooling curves for the sheared and non-sheared AZ91 alloy melt. The cooling rate used in the calculation is $0.22^{\circ} \mathrm{C} / \mathrm{s}$ for the sheared melt and $0.2{ }^{\circ} \mathrm{C} / \mathrm{s}$ for the non-sheared melt. The calculated $\Delta T_{\max }$ is $0.71^{\circ} \mathrm{C}$ and $0.99^{\circ} \mathrm{C}$ for the sheared and non-sheared melt, respectively, which are in good agreement with the corresponding experimental results.

Fig. 8 The theoretically predicted $T_{\mathrm{f}}$ (a) and solid fraction at $T_{\mathrm{f}}(\mathrm{b})$ as a function of cooling rate in the sheared and non-sheared melt. $\sigma=0.45$ and $d_{0}=0.07 \mu \mathrm{m}$ were used for the calculation. $T_{\mathrm{f}}$ 
corresponds to the temperature at which the nucleation finished due to the recalescence. The sheared melt always exhibits higher $T_{\mathrm{f}}$ than that of the non-sheared melt at the same cooling rate.

Fig. 9 The theoretically calculated number density of active nucleating particles (a) and grain size (b) in the sheared and non-sheared melt as a function of cooling rate. 
Figures:

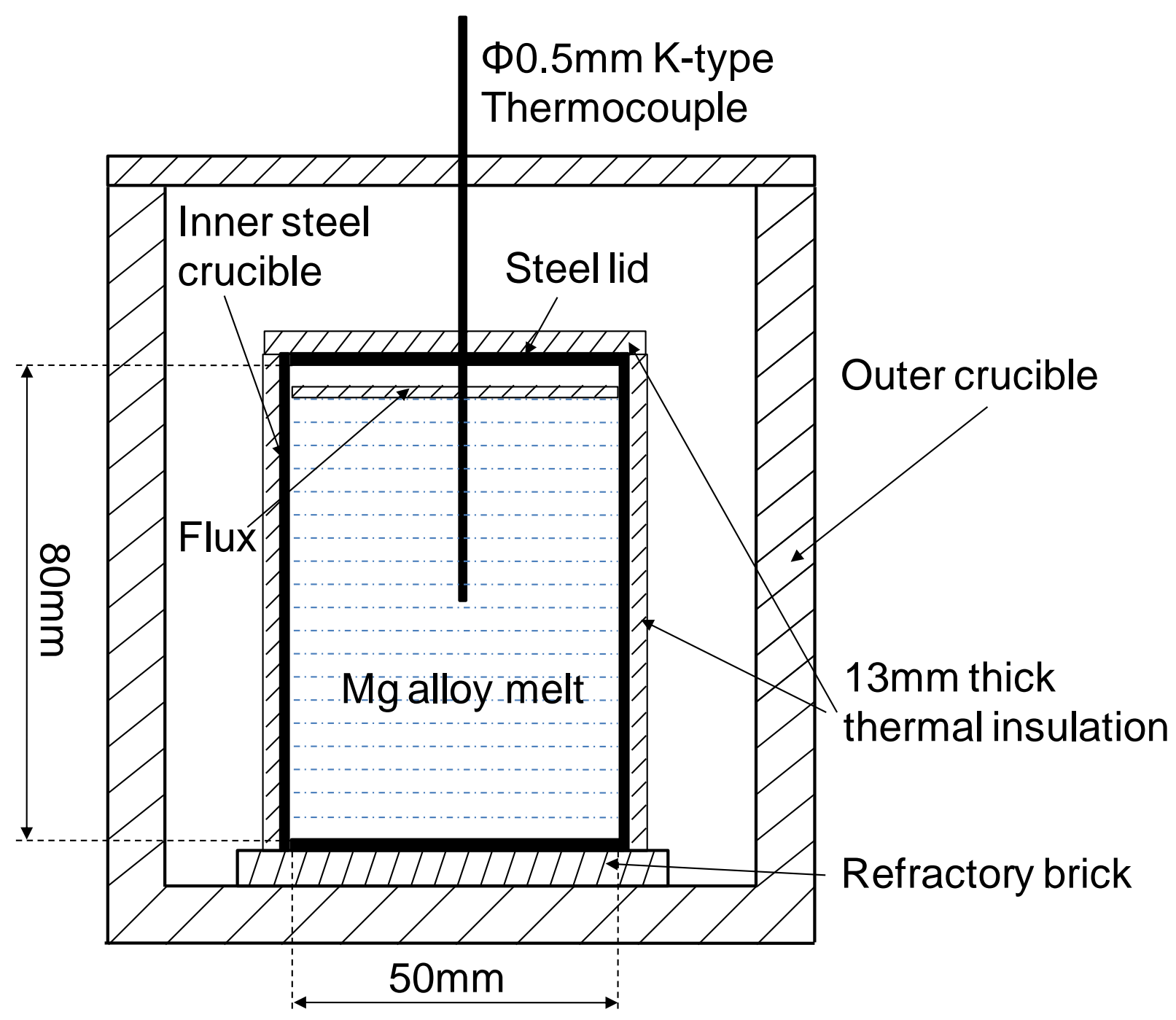

Fig. 1 


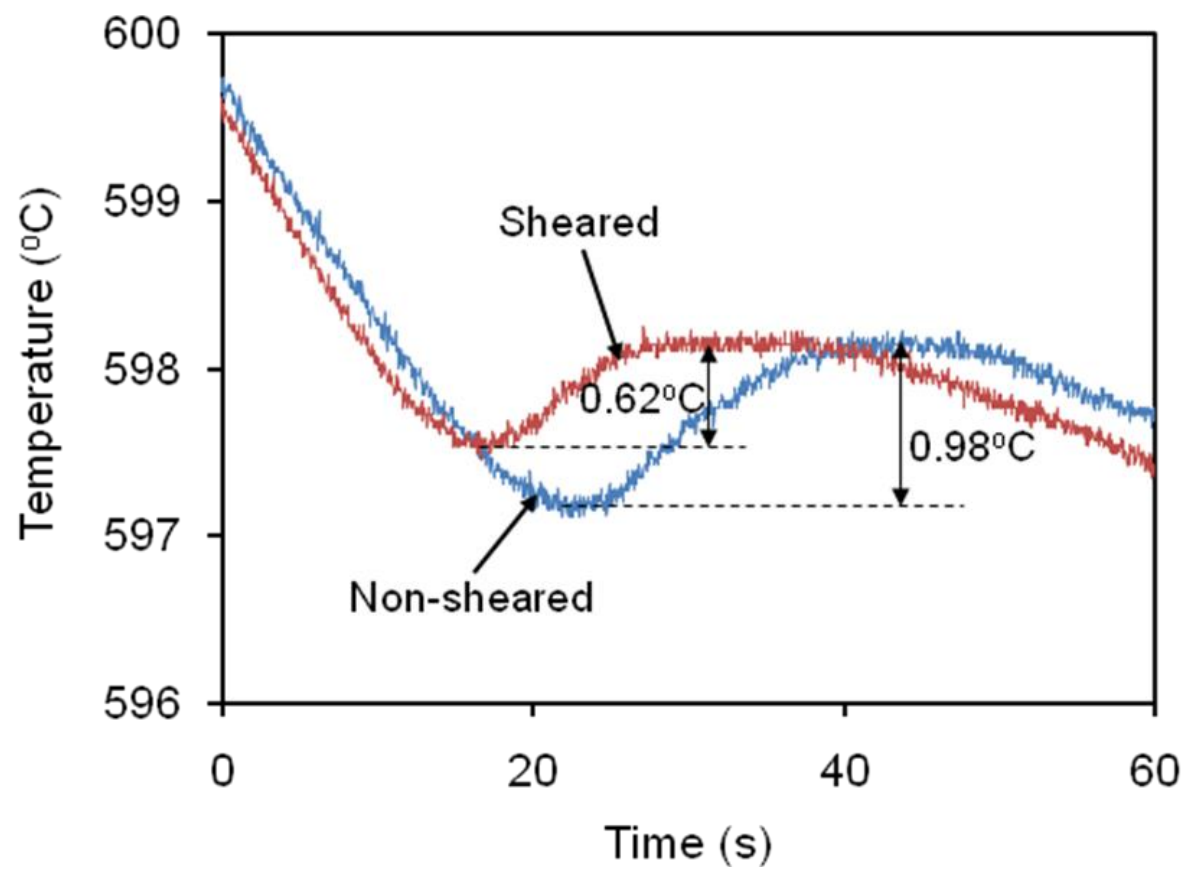

Fig.2 


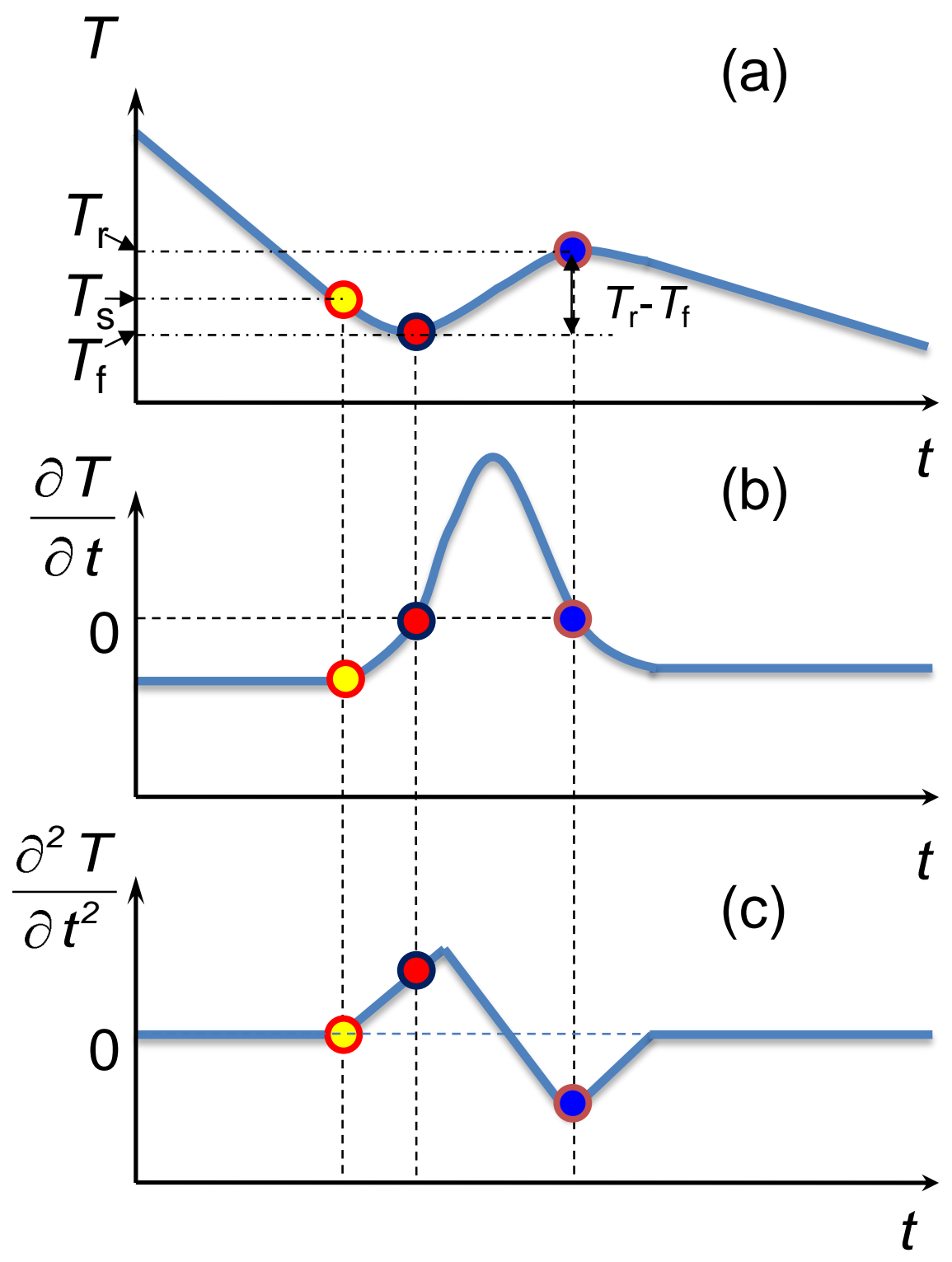

Fig.3 

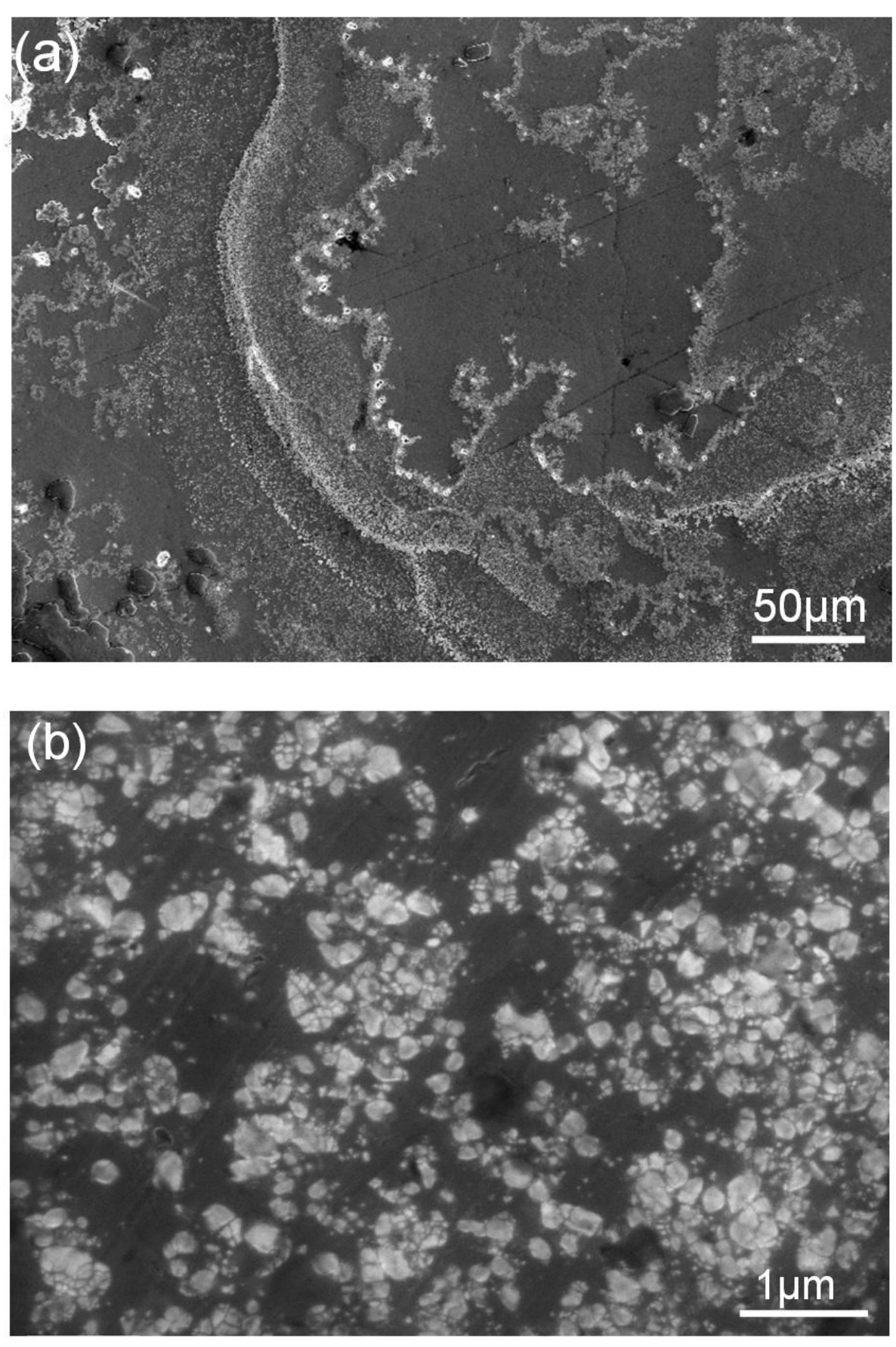

Fig.4 


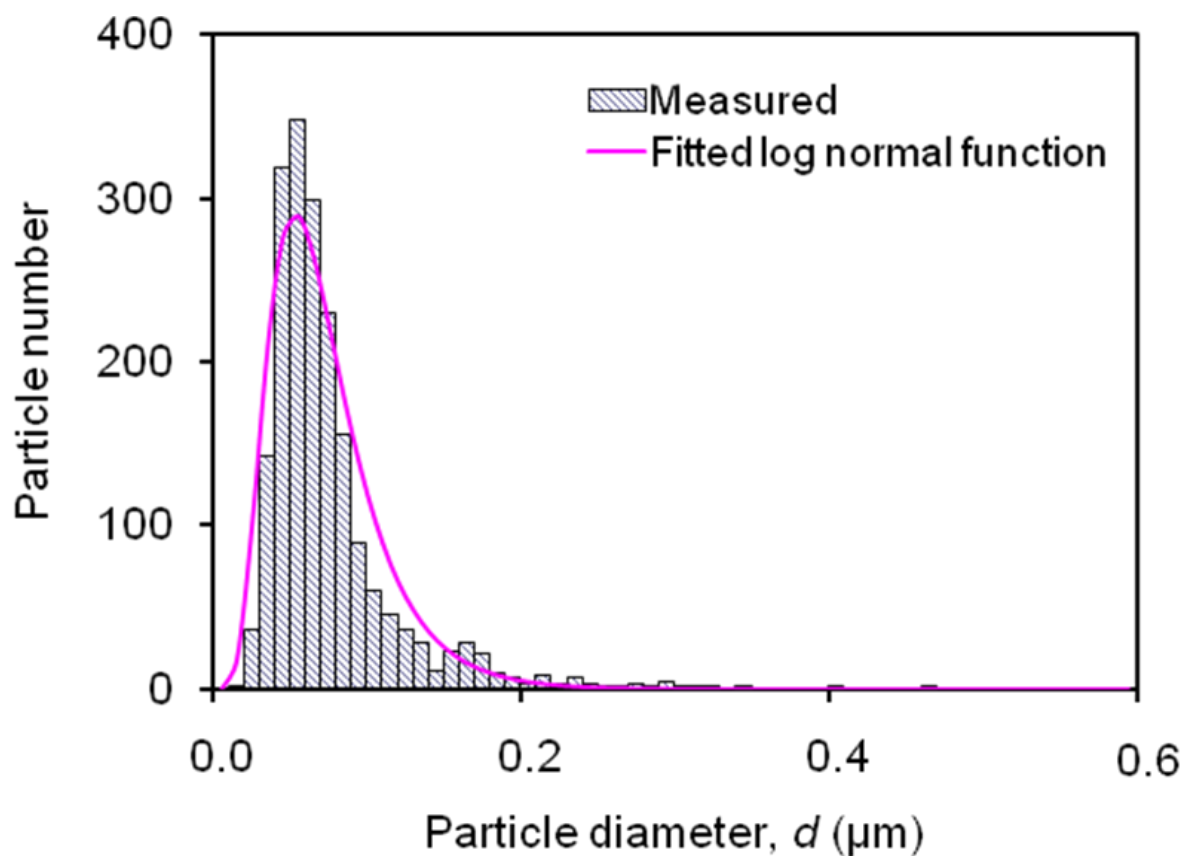

Fig.5 

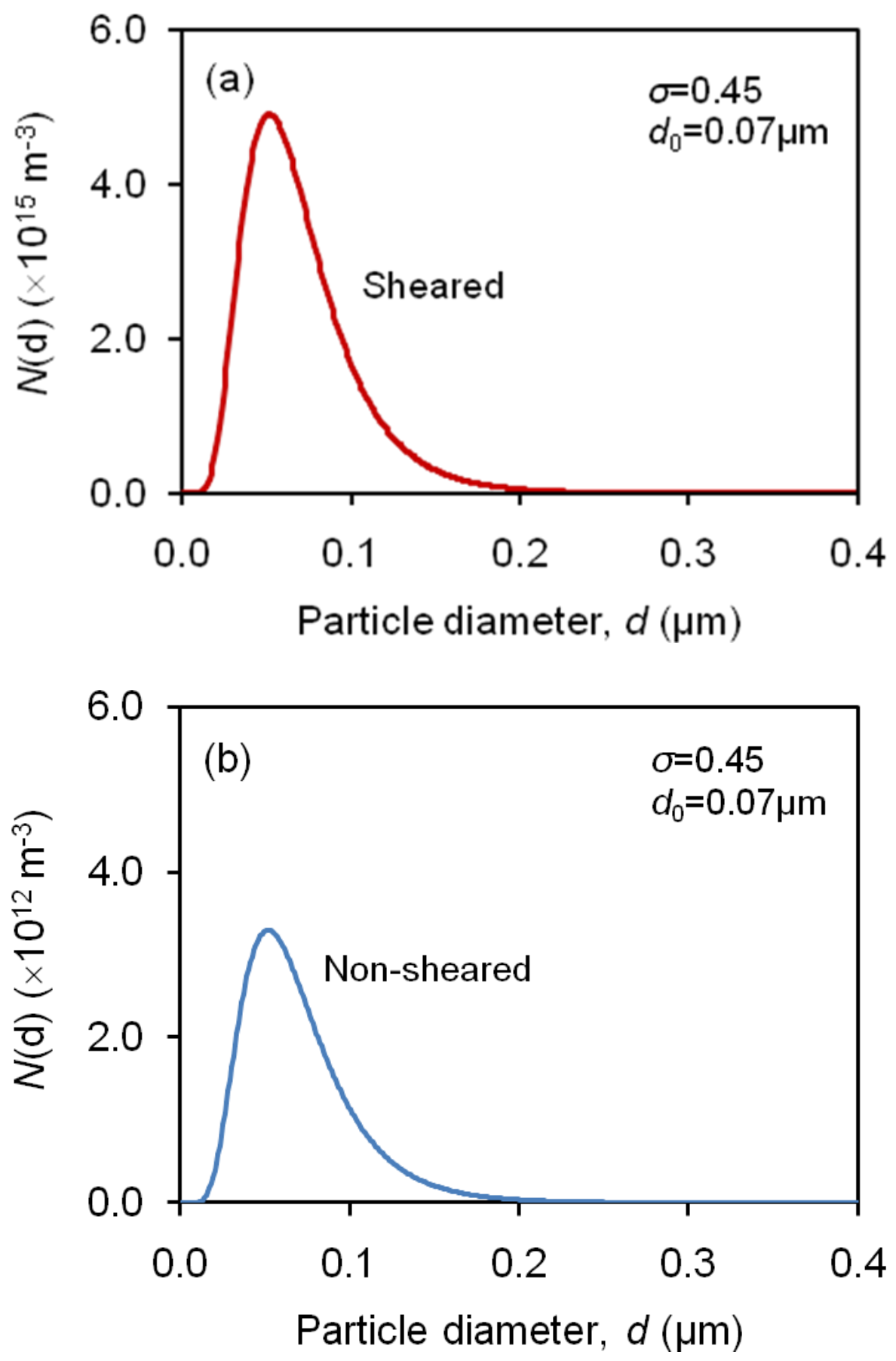

Fig.6 


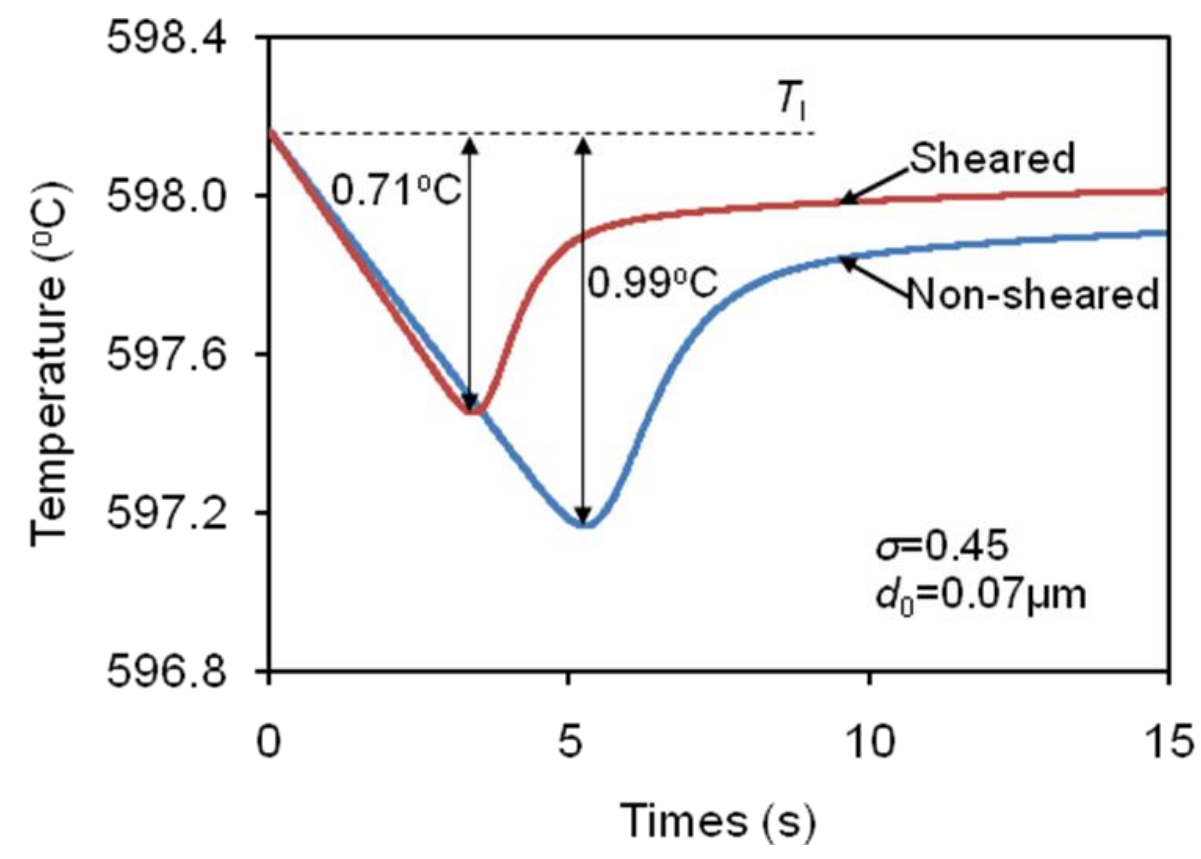

Fig. 7 

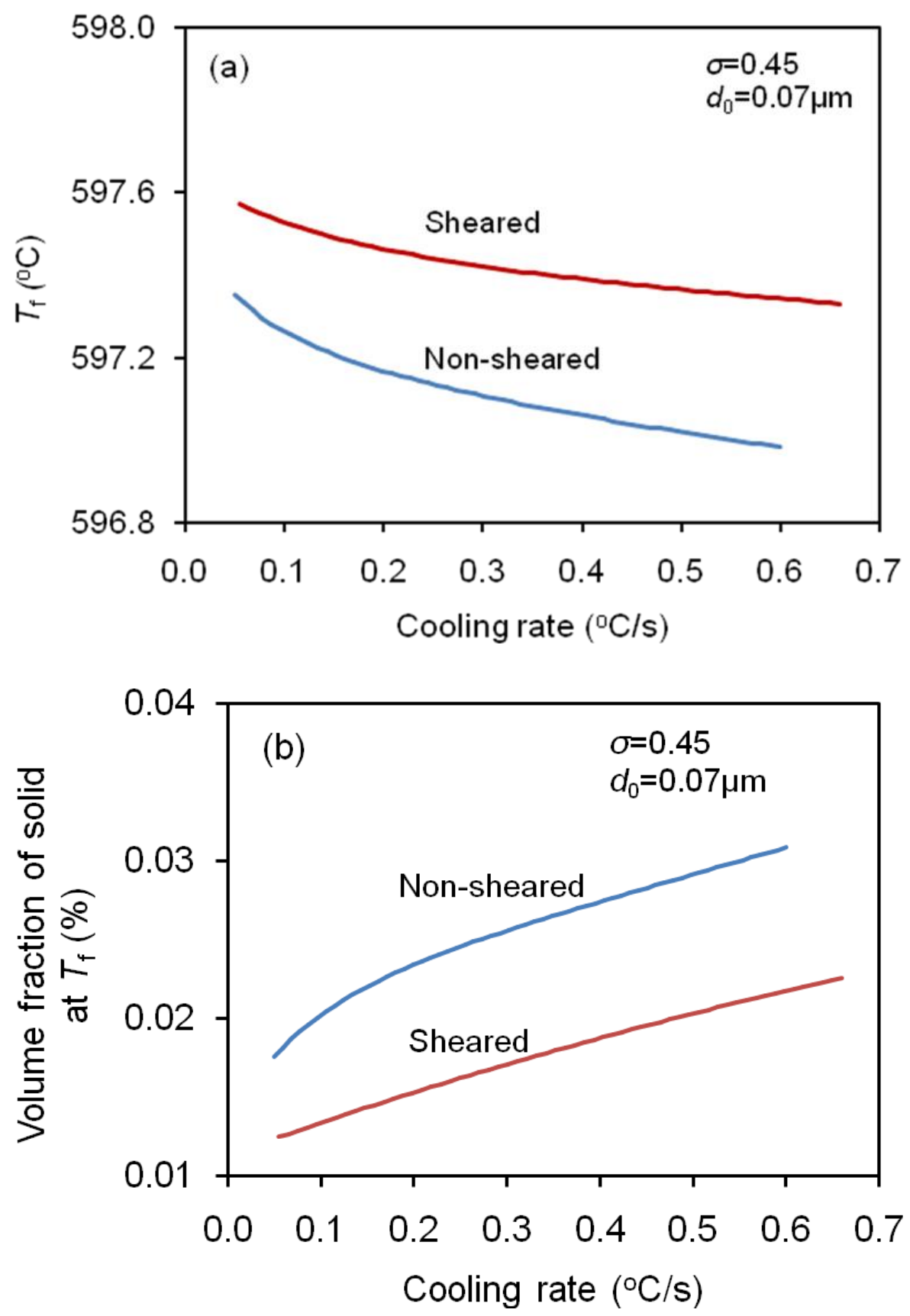

Fig. 8 


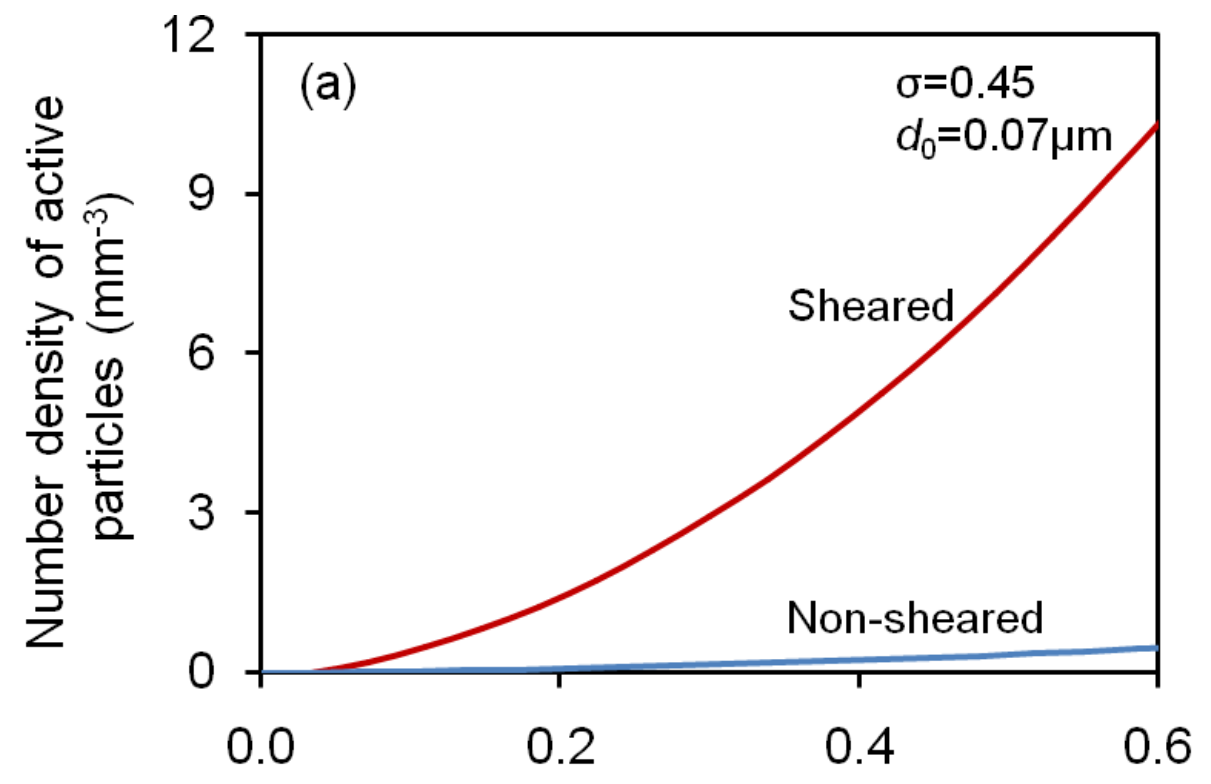

Cooling rate $\left({ }^{\circ} \mathrm{C} / \mathrm{s}\right)$

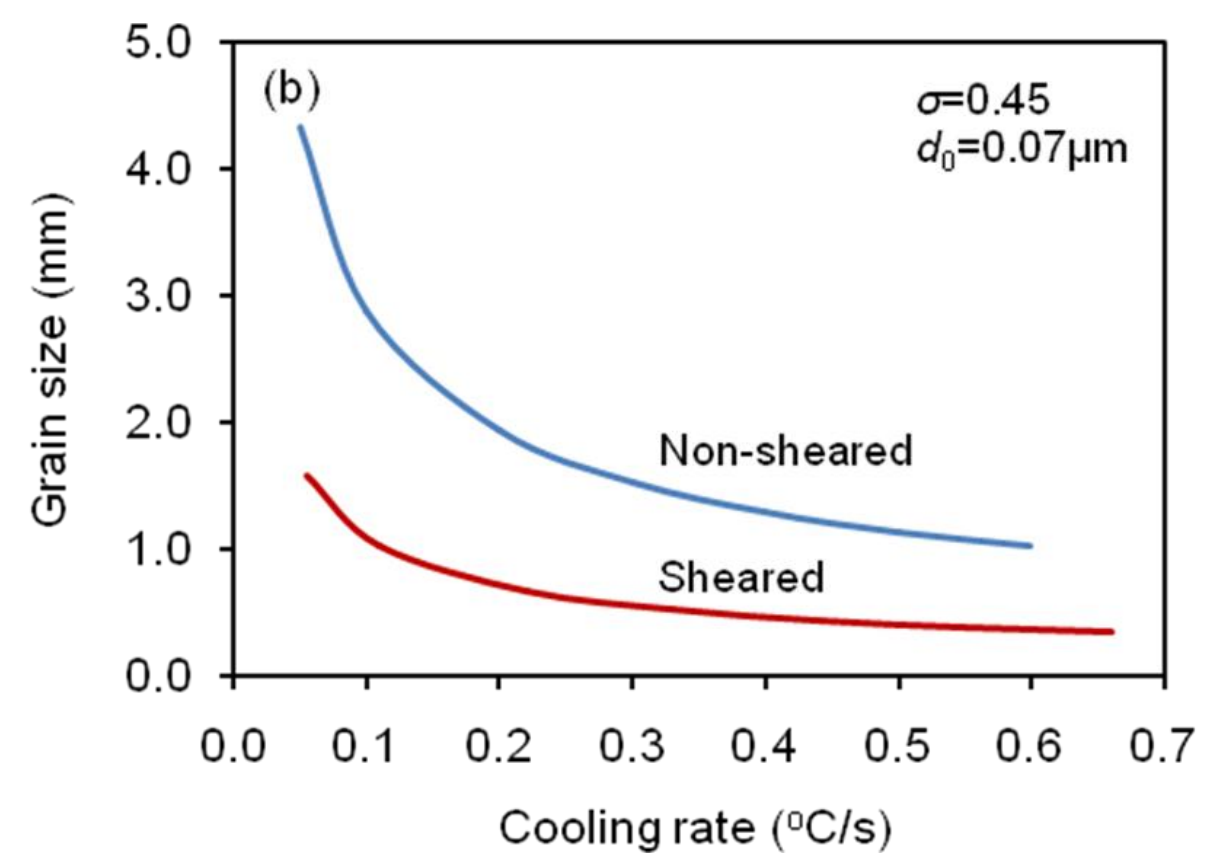

Fig. 9 